\title{
LA-UR-95:- 2878
}

Los Alamos National Laboratory is operated by the University of California for the United States Department of Energy under contract W-7405-ENG-36.

TITLE: THE LINEAR ALGEBRAIC METHOD FOR ELECTRON-MOLECULE COLLISIONS

AUTHOR(S): Lee A. Collins and Barry I. Schneider

PRESENTED AT: Comparative Study of Current Methodologies in Electron-Molecule Scattering, Cambridge, MA, March 11-13, 1993

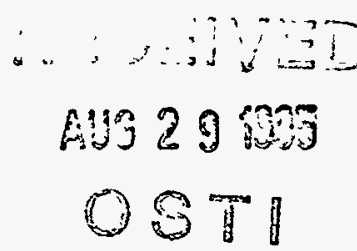

DISTRIBUTION OF THIS DOCUMENT IS UNLIMITED

By acceptance of this article, the published recognizes that the U.S. Government retains a non-exclusive, royalty-free license to publish or reproduce the published form this contribution, or to allow others to do so, for U.S. Government purposes.

The Los Alamos National Laboratory requests that the publisher identify this article s work performed under the auspices of the U.S. Department of Energy.

\section{DISCLAIMER}

This report was prepared as an account of work sponsored by an agency of the United States Government. Neither the United States Government nor any agency thereof, nor any of their employees, makes any warranty, express or implied, or assumes any legal liability or responsibility for the accuracy, completeness, or usefulness of any information, apparatus, product, or process disclosed, or represents that its use would not infringe privately owned rights. Reference herein to any specific commercial product, process, or service by trade name, trademark, manufacturer, or otherwise does not necessarily constitute or imply its endorsement, recommendation, or favoring by the United States Government or any agency thereof. The views and opinions of authors expressed herein do not necessarily state or reflect those of the United States Government or any agency thereof.

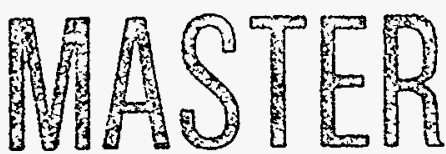




\section{DISCLAIMER}

Portions of this document may be illegible in electronic image products. Images are produced from the best available original document. 


\title{
THE LINEAR ALGEBRAIC METHOD FOR ELECTRON-MOLECULE COLLISIONS
}

\author{
Lee A. Collins ${ }^{1}$ and Barry I. Schneider ${ }^{2}$ \\ 1 Theoretical Division, Group T-4, Los Alamos National Laboratory, Los \\ Alamos, NM 87545 \\ 2 Physics Division, National Science Foundation, Arlington, VA 22230
}

\section{BASIC CONCEPTS}

In order to find numerical solutions to many problems in physics, chemistry and engineering it is necessary to place the equations of motion (classical or quantal) of the variables of dynamical interest on a discrete mesh. The formulation of scattering theory in quantum mechanics is no exception and leads to partial differential or integral equations which may only be solved on digital computers. Typical approaches introduce a numerical grid or basis set expansion of the scattering wavefunction in order to reduce the problem to the solution of a set of algebraic equations. Often it is more convenient to deal with the scattering matrix or phase amplitude rather than the wavefunction but the essential features of the numerics are unchanged.

In this section we will formulate the Linear Algebraic Method (LAM) for electronatom/molecule scattering for a simple, one-dimensional radial potential ${ }^{1-2}$. This will illustrate the basic approach and enable the uninitiated reader to follow the subsequent discussion of the general, multi-channel, electron-molecule formulation without undue difficulty. We begin by writing the Schroedinger equation for the s-wave scattering of a structureless particle by a short-range, local potential.

$$
-\frac{1}{2} \frac{d^{2} \Psi}{d r^{2}}+(V(r)-E) \Psi(r)=0
$$

By re-writing this equation in its integral form,

$$
\Psi(r)=\Psi_{0}(r)+\int d r^{\prime} G\left(r \mid r^{\prime}\right) V\left(r^{\prime}\right) \Psi\left(r^{\prime}\right)
$$

where

$$
\begin{aligned}
\Psi_{0}(r) & =\sin (\mathrm{k} r) \\
G\left(r \mid r^{\prime}\right)=\text { Green's function } & =-2 \frac{\sin \left(\mathrm{k} r_{<}\right) \cos \left(\mathrm{k} r_{>}\right)}{\mathrm{k}} \\
\mathrm{k} & =\sqrt{2 \mathrm{~F}_{1}}
\end{aligned}
$$


it becomes straightforward to irarporate the two physical boundary conditions required of a standing wave solution to the scattering equation, regularity at the origin and incident free wave $\sin (\mathrm{kr})$ plus outgoing $\cos (\mathrm{kr})$ behavior at large distances from the scatterer. We now proceed by introducing a numerical quadrature with points $r_{i}$ and weights $w_{i}$ into the integral and then set the co-ordinate $r$ to one of the quadrature points. This yields,

$$
\Psi\left(r_{i}\right)=\Psi_{0}\left(r_{i}\right)+\sum_{j} G\left(r_{i} \mid r_{j}\right) w_{j} V\left(r_{j}\right) \Psi\left(r_{j}\right)
$$

By defining vector and matrix elements as,

$$
\begin{aligned}
{[\Psi]_{\mathbf{i}} } & =\Psi\left(r_{i}\right) \\
{\left[\Psi_{0}\right]_{\mathbf{i}} } & =\Psi_{0}\left(r_{i}\right) \\
{[\tilde{\mathbf{V}}]_{\mathbf{i j}} } & =w_{i} V\left(r_{i}\right) \delta_{i, j} \\
{[G]_{i j} } & =G\left(r_{i} \mid r_{j}\right)
\end{aligned}
$$

it is possible to write $\mathrm{Eq}(3)$ in the matrix notation,

$$
\begin{aligned}
\mathbf{M} \Psi & =\Psi_{0} \\
\mathbf{M} & =\mathbf{I}-\mathbf{G} \tilde{\mathbf{V}}
\end{aligned}
$$

as a set of linear algebraic ( LA ) equations for the unknown vector $\boldsymbol{\Psi}$. These may be solved using Gaussian elimination or a number of other standard linear systems packages $^{3}$ which are readily available on most computers. If the matrix GV becomes too large to be held in central memory, there are iterative techniques ${ }^{4}$ which may be employed to effect a solution of $\mathrm{Eq}$ (3). One such approach, the variation-iteration method, will be discussed in much greater detail in a subsequent section. The great advantage of the integral equation formulation, which becomes much more apparent in the multichannel scattering problem, is the numerical stability associated with the incorporation of the two point boundary conditions in the propagator, G. This allows weakly open and closed (exponentially decaying ) channels to be calculated in a stable fashion, a much more difficult process to accomplish via the propagation of the solution of a ( set of ) differential equation $(s)^{5}$.

\section{GENERAL FOMALISM}

In this section we will formulate the general $\mathrm{LAM}^{6-16}$ for a wavefunction which is expanded as a sum of a close-coupled plus correlation term (CCC) ${ }^{17}$. In addition, we restrict ourselves to the fixed nuclei approximation; all rotational and vibrational degrees of freedom will be ignored and the molecule will be taken to be fixed in space. Rotational and vibrational motion away from thresholds and resonances may be treated using the adiabatic nuclei formalism ${ }^{19-20}$. When that is inappropriate, off-shell techniques or extensions of the close-coupled form of the equations to include rotations and vibrations may be invoked. These are treated in other chapters of this volume ${ }^{17}$ and their inclusion here would add little to the present discussion. The CCC wavefunction may be expressed as,

$$
\Psi\left(1,2, \cdots, n_{T+1}\right)=\sum \Theta_{c}\left(1,2, \cdots, n_{T}\right) f_{c}\left(n_{T+1}\right)+\sum \Xi_{q}\left(1,2, \cdots, n_{T+1}\right) D_{q}
$$


where $\Theta_{c}$ are the channel wavefunctions and $\Xi_{q}$ the correlation terms. The te sachannel wavefunction is used here to denote the product of an internal electronic state of the target times an overall spin eigenfunction of the composite system. This quite general form to the wavefunction allows us treat all desired open channels explicitly and to incorporate the effects of closed channels via an optical potential and/or by the explicit introduction of real or pseudo states as with the open channels. The optical potential results from using Feshbach ${ }^{21}$ partitioning techniques to formally remove the second sum in favor of a non-local, energy-dependent potential. It will be assumed that the optical potential is capable of an $\mathrm{N}$-term separable expansion in terms of a set of square integrable functions. Under these rather general assumptions it is possible to reduce the scattering problem to a set of coupled integro-differential equations of the form,

$$
\left(-\frac{1}{2} \nabla^{2}-\epsilon_{c}\right) f_{c}(r)+\sum_{c^{\prime}} \int d r^{\prime} V_{c, c^{\prime}}\left(r, r^{\prime}\right) f_{c^{\prime}}\left(r^{\prime}\right)=0
$$

where the potential, $V_{c, c^{c}}$, consists of a local(l) and non-local(nl) part, i.e.,

$$
V_{c, c^{\prime}}\left(r, r^{\prime}\right)=\delta\left(r-r^{\prime}\right) V_{c, c^{\prime}}^{l}(r)+V_{c, c^{\prime}}^{n l}\left(r, r^{\prime}\right)
$$

and the non-local potential is expanded as,

$$
V_{c, c^{\prime}}^{n l}\left(r, r^{\prime}\right)=\sum_{\alpha} \sum_{\beta} \phi_{c, \alpha}(r) V_{c \alpha, c^{\prime} \beta} \phi_{c^{\prime}, \beta}\left(r^{\prime}\right)
$$

The local potential includes the attractive interaction of the scattering electron with the nuclei and the direct $\left(c=c^{\prime}\right)$ or transition $\left(c \neq c^{\prime}\right)$ electronic potential arising from the repulsive electron-electron interactions. At large electron-molecule distances the local potential has a typical multi-polar form, reflecting the permanent and transition moments of the charge distributions of the target. The non-local interaction arises from the requirement of overall antisymmetry of the scattering wavefunction to exchange of the scattering electron with any of the bound electrons of the target and from the optical potential. Typical exchange interactions are short range and vanish exponentially at large electron-molecule separations. The optical potential reflects the moments induced by the scattering electron in the charge cloud of the target as well as shorter range, exchange terms. In the original LAM, Eq (5) is further expanded in a set of spherical harmonics and the three dimensional, coupled equations are replaced by an even larger set of coupled, radial equations. If we use the symbol $\gamma$ to represent the composite label $(\mathrm{c}, \mathrm{l}, \mathrm{m})$, where $(1, \mathrm{~m})$ are the quantum numbers of the spherical harmonic used in the expansion, it is possible to transform the set of coupled radial integro-differential equations into the following set of coupled integral equations by utilizing the Green's function for free-particle motion,

$$
f_{\gamma}^{\gamma^{\prime \prime}}(r)=\delta_{\gamma, \gamma^{\prime \prime}} f_{\gamma}^{0}(r)+\sum_{\gamma^{\prime}} \int d r^{\prime} d r^{\prime \prime} G_{\gamma}^{0}\left(r, r^{\prime}\right) V_{\gamma, \gamma^{\prime}}\left(r^{\prime}, r^{\prime \prime}\right) f_{\gamma^{\prime}}^{\gamma^{\prime \prime}}\left(r^{\prime \prime}\right) .
$$

where the label $\gamma^{\prime \prime}$ is used to denote the linearly independent solution having its incident wave in channel $\gamma^{\prime \prime}$. By introducing a quadrature into the integrals over $r$ and $r^{\prime}$, it is possible to convert the entire set of coupled integral equations into a larger set of linear algebraic equations defined by the composite index $\Gamma=(c, l, m, i)$, where $\gamma=(c, l, m)$ specifies the channel and $i$ the quadrature point of the quadrature. Thus, assuming there are $N_{\gamma}$ channels, any matrix element may be located using its $\gamma$ and $i$ index as,

$$
\Gamma=\left(N_{\gamma}-1\right) \gamma+i
$$


This enables us to regard the vectars (matrices) as a set of supervectors (matrices) labelled by channel indices. The running index of each sub-block is the set of quadrature points. Proceeding in this fashion, we may define the elements of our vectors and matrices as,

$$
\begin{aligned}
{[\mathbf{f}]_{\Gamma} } & =f_{\gamma}\left(r_{i}\right) \quad\left[\mathbf{f}^{0}\right]_{\Gamma}=f_{\gamma}^{0}\left(r_{i}\right) \\
{[\mathrm{M}]_{\Gamma, \Gamma,} } & =\delta_{\gamma, \gamma^{\prime}}-\sum_{k} G_{\gamma}^{0}\left(r_{i}, r_{k}\right) w_{k} V_{\gamma, \gamma^{\prime}}\left(r_{k}, r_{j}\right) w_{j}
\end{aligned}
$$

which enables us to write the entire set of equations in the compact notation,

$$
\mathbf{M f}=\mathbf{f}^{\mathbf{0}}
$$

Just as in the simple, one-dimensional example discussed earlier, the LA formulation has the advantage of numerical stability and conceptual simplicity. The disadvantage is that the practitioner must often deal with large sets of linear algebraic equations. The solution of these equations can be computationally and memory intensive. One useful approach to reduce the size of these equations is to formulate the LA problem inside a sphere sufficiently large to enclose the exchange/correlation region of the scatterer and to use more standard propagation techniques beyond that radius ${ }^{16}$. This philosophy, which is formally identical to the R-matrix method, allows us to treat the short-range, non-local interactions using the LAM inside the sphere and to integrate coupled, differential equations outside the sphere. The only modification required is to alter the boundary conditions imposed on the Green's function from outgoing wave to some ( usually zero ) log-derivative condition on the R-matrix surface. The form of the LA equations does not change. In essence, one is solving the integral equation for the full, interacting Green's function, which is equal to the R-matrix when evaluated on the surface of the sphere. This $\mathrm{R}$-matrix may then be propagated to very large distances via $\mathrm{R}$-matrix propagation ${ }^{22-23}$, continued fraction ${ }^{24}$ or other approaches which are very efficient for coupled channel, local interactions.

\section{NUMERICAL TECHNIQUES}

\subsection{General Remarks}

Numerical techniques for the solution of the LA equations fall into two classes: 1) off-the-shelf methods which reduce the LA coefficient matrices to specialized forms (i.e. LU factorizations, Gaussian elimination, etc.) and 2) specialized iterative techniques. The off-the-shelf methods almost always work and are certainly to be preferred when the matrices are small enough to fit in core or are rot particularly well behaved. The work to solve the LA equations using such techniques goes like $N^{3}$ where $N$ is the size of the matrix. This is to be contrasted with iterative techniques which are dominated by matrix-vector multiplies and behave like $N^{2} M$ where $M$ is the number of iterations needed to achieve convergence. If $M$ can be kept much smaller than $N$, the iterative approach can be quite practical and valuable. A further advantage of iterative techniques is that they may be easily developed to take advantage of any special structure to the matrices in performing the computationally intensive matrix-vector multiplies. For the problem under consideration, there are considerable simplifications in both computational effort and storage requirements resulting from developing a rapidly converging iterative approach. This has been done by the authors ${ }^{11-12}$ and it is described in some 


\subsection{Variation-Iteration Method}

Iterative methods have a long history in numerical analysis. The Jacobi and GaussSeidel methods ${ }^{4}$ are described in almost all textbooks dealing with iterative treatments of linear equations. In all of these methods one begins with some guess to the solution of the set of LA equations and then computes either a correction to that guess or what is equivalent, an updated solution. The process is continued until there is (essentially) no change in the next member of the iteration sequence. However, there is no guarantee that this process will converge. The question of convergence or the lack of it can often be traced to the eigenvalue spectrum of the iteration matrix. The iteration process is equivalent to expanding $(\mathbf{I}-\mathbf{G V})^{-1}$ in a power series in $\mathbf{G V}$ and is only justified if the eigenvalues of GV lie inside the unit circle. However, it is possible to combine iterative techniques with variational methods to produce convergent results even when there are eigenvalues outside the unit circle. In essence the variational step sums the geometric series by solving a "small" set of algebraic equations which results from the projection of the full set of LA equations into the space of the iterates. Stated somewhat differently, the iterates are used as a set of basis vectors for the expansion of the unknown solution. The expansion coefficients are determined either by invoking a variational principle or by projecting the full set of LA equations onto the iterate space. If the iterates are a good basis for the expansion of the unknown solution to the LA equations, convergence should be rapid. However, as long as one can develop $N$ linearly independent vectors over the course of the iteration process, one will eventually have enough flexibility to span the space of the $N$ dimensional vectors of the full LA equations. In practice, if convergence is slow, there is a reasonable probability that the iteration sequence will produce linear dependence of the expansion vectors and this can bring the entire procedure to a grinding halt. The variation-iteration process may be initiated using any starting vector. Typically we use the "normalized" Born approximation, defined as,

$$
\begin{aligned}
\mathbf{v}^{0} & =\frac{\mathbf{f}^{\mathbf{0}}}{\left\langle\mathbf{f}^{\mathbf{0}} \mid \mathbf{f}^{0}\right\rangle^{\frac{2}{2}}} \\
\left\langle\mathbf{f}^{0} \mid \mathbf{f}^{0}\right\rangle & =\sum_{\gamma, i} f_{\gamma}^{0}\left(r_{i}\right) w_{i} f_{\gamma}^{0}\left(r_{i}\right)
\end{aligned}
$$

to begin the process. We then initiate the following sequence:

1. At the $n^{\text {th }}$ step in the iteration process, compute an iterate via the formula,

$$
\mathrm{f}^{\mathrm{n}}=\mathbf{M} \mathbf{v}^{\mathrm{n}-1}
$$

2. Expand the solution to the LA equations as,

$$
\mathrm{f}=\sum_{i=1}^{n} C_{i} \mathbf{v}^{\mathbf{i}-1}
$$

3. Determine the $C_{i}$ by substituting the expansion of step 2 into Eq ( 11$)$ and then projecting onto the set of $\mathbf{v}$. This yields,

$$
C_{i}=\left\langle\mathrm{v}^{\mathrm{i}-1} \mid \mathrm{f}^{0}\right\rangle+\sum_{j=1}^{n}\left\langle\mathrm{v}^{\mathrm{i}-1} \mid \mathrm{f}^{\mathrm{j}}\right\rangle C_{j}
$$

a "small" set of equations which may be solved by standard techniques. Once the $C_{i}$ have been calculated, it is possible to compute the RMS error to the set of LA equations. 
If the RMS error falls below the desired. level, the process is stopped.

4. If that is not the case, a new vector is computed using the Gram-Schmidt process.

$$
\begin{aligned}
& \mathbf{T}^{\mathbf{n}}=\mathbf{f}^{\mathbf{n}}-\sum_{j=1}^{n} \mathbf{v}^{\mathbf{j}-1}\left\langle\mathbf{v}^{\mathbf{j}-1} \mid \mathbf{f}^{\mathbf{n}}\right\rangle \\
& \mathbf{v}^{\mathbf{n}}=\frac{T^{\mathbf{n}}}{\left\langle\mathbf{T}^{\mathbf{n}} \mid \mathbf{T}^{\mathbf{n}}\right\rangle^{\frac{1}{2}}}
\end{aligned}
$$

and then return to step 1 . The vectors and iterates which form the basis of this procedure are called a Krylov sequence ( KS ). The KS is at the heart of most iterative approaches to the solution of large linear systems and eigenvalue problems. The differences between the various methods such as the Arnoldi, Generalized Minimum Residue, and Lanczos approaches is in how these quantities are manipulated to get the final information needed. The interested reader should consult the references for details ${ }^{18.4}$. The main concern is how to make the calculation of the matrix-vector multiply as efficient as possible. Fortunately, the quasi-separable structure of the Lippmann-Schwinger kernel, facilitates some simplification. To see this in more detail, we specialize Eq ( 13 ) to the scattering problem.

$$
f_{\gamma}\left(r_{i}\right)=\sum_{\gamma^{\prime}} \sum_{j, k} G_{\gamma}^{0}\left(r_{i}, r_{j}\right) w_{j} V_{\gamma, \gamma^{\prime}}\left(r_{j}, r_{k}\right) w_{k} v_{\gamma^{\prime}}\left(r_{k}\right)
$$

If we define $N_{c}$ as the number of channels, $N_{b}$ as the number of basis functions, $N_{p}$ as the number of quadrature points, and the intermediate vector,

$$
J_{\gamma}\left(r_{i}\right)=\sum_{\gamma^{\prime}} \sum_{j} V_{\gamma, \gamma^{\prime}}\left(r_{i}, r_{j}\right) w_{j} v_{\gamma^{\prime}}\left(r_{j}\right)
$$

the work required to construct $J_{\Gamma}$ goes like $N_{\gamma}^{2} N_{p}$ for a local potential and as the greater of the products $N_{\gamma} N_{b} N_{p}$ or $N_{\gamma}^{2} N_{b}^{2}$ for a non-local, separable potential. Once $J_{\Gamma}$ is formed we compute,

$$
f_{\gamma}\left(r_{i}\right)=\sum_{j} G_{\gamma}^{0}\left(r_{i}, r_{j}\right) w_{j} J_{\gamma}\left(r_{j}\right)
$$

Since the Green's function may be written as,

$$
\begin{aligned}
G_{\gamma}^{0}\left(r, r^{\prime}\right) & =-2 \frac{\mathrm{j}\left(\mathrm{k}_{\gamma} r_{<}\right) \eta\left(\mathrm{k}_{\gamma} r_{>}\right)}{\mathrm{k}_{\gamma}} \\
\mathrm{k}_{\gamma} & =\sqrt{2 \epsilon_{\gamma}} \\
\mathrm{j}(\mathrm{k} r) & =\text { Bessel Function } \quad \eta(\mathrm{k} r)=\text { Neumann Function }
\end{aligned}
$$

it is possible to write the sum over the quadrature points as,

$$
f_{\gamma}\left(r_{i}\right)=\eta_{\gamma}\left(\mathrm{k}_{\gamma} r_{i}\right) \sum_{j=1}^{i} \mathrm{j}_{\gamma}\left(\mathrm{k}_{\gamma} r_{j}\right) w_{j} J_{\gamma}\left(r_{j}\right)+\mathrm{j}_{\gamma}\left(\mathrm{k}_{\gamma} r_{i}\right) \sum_{j=i+1}^{n} \eta_{\gamma}\left(\mathrm{k}_{\gamma} r_{j}\right) w_{j} J_{\gamma}\left(r_{j}\right)
$$

From the basic definitions, the following relationships arise,

$$
\begin{aligned}
\sum_{j=1}^{i} \mathrm{j}_{\gamma}\left(\mathrm{k}_{\gamma} r_{j}\right) w_{j} J_{\gamma}\left(r_{j}\right) & =\sum_{j=1}^{i-1} \mathrm{j}_{\gamma}\left(\mathrm{k}_{\gamma} r_{j}\right) w_{j} J_{\gamma}\left(r_{j}\right)+\mathrm{j}_{\gamma}\left(\mathrm{k}_{\gamma} r_{i}\right) w_{i} J_{\gamma}\left(r_{i}\right) \\
\sum^{n} \eta_{\gamma}\left(\mathrm{k}_{\gamma} r_{j}\right) w_{j} J_{\gamma}\left(r_{i}\right) & =\sum^{n} \eta_{\gamma}\left(\mathrm{k}_{\gamma} r_{j}\right) w_{j} J_{\gamma}\left(r_{i}\right)+\eta_{\gamma}\left(\mathrm{k}_{\gamma} r_{i+1}\right) w_{i+1} J_{\gamma}\left(r_{i+1}\right)
\end{aligned}
$$


Thus the forward and backward susamations over quadrature points may be performad. using recursion relations involving "one" matrix muliply per step. It should be noted that the effort required to perform the indicated recursions is identical to that of most propagation approaches. By performing the operation in the forward ( backward) direction for the first ( second ) of Eq (22), we can insure the numerical stability of the recursion scheme. Standard propagation methods for the integration of the coupled differential or integral equations of scattering theory rely totally on either forward or backward integration. This is required since the solution must be known at some subset of prior points before it can "advanced". In contrast, the iterative approach always deals with an integrand which is known at all points in space. This enables the practitioner to integrate in both directions and to thus ensure numerical stability without elaborate stabilization procedures.

\subsection{Further Developments}

The current implementation of the LAM relies heavily on single-center expansions to reduce the three-dimensional equations to a larger set of one-dimensional equations. A serious disadvantage of this approach is the need to expand a singular, Coulomb potential away from its natural center. This often forces the spherical harmonic expansion to be quite slowly convergent, leading to linear systems of relatively large dimension. In addition, the idea of expanding an inherently multi-center charge distribution about a single co-ordinate system, is physically unappealing. Some recent developments, which can only be sketched here, will allow us to remove this limitation. At the core of the new approach is the recent multi-center integration scheme of Becke ${ }^{25}$, already being successfully applied in local density functional calculations in quantum chemistry. The central idea is to define local, i.e. atomic, numerical grids and to then weight these such that any three-dimension integral will be reproduced by the sum of the atomic integrals. The weighting function for each atomic grid is chosen to be unity at the atomic nucleus and to go smoothly to zero at any other nucleus. By experimentation, Becke found a practical functional form which achieves the desired purpose in an efficient fashion. In a subsequent paper, Becke ${ }^{26}$ extended these ideas to the solution of the Poisson equation. Here he suggested the use of local spherical harmonic expansions combined using the weighting ideas of his first paper. Although the specifics of his numerical approach to the radial equations need not concern us here, it is worth pointing out that there are efficient, integral equation approaches to this problem which have been developed to solve scattering problems. We have already adapted some of these ideas to the Poisson equation and are now examining the inhomogeneous wave equation which forms the basis of our variation-iteration method. We are confident that we will be able to use this multi-center approach to effect a totally numerical solution to the electron-polyatomic scattering problem. Research along these lines is already in progress and we expect to report some preliminary results quite soon.

\section{ELECTRON-ATOM SCATTERING IN INTENSE FIELDS}

Physical phenomena abound for which the linear algebraic approach provides an effective means of explication. Those cases involving scattering of heavy or light particles are easily discerned. However, other classes of atomic and molecular processes lend themselves to the technique. One particular example on which we shall concentrate concerns an atom subjected to an intense electromagnetic radiation field. We demonstrate that the basic Schroedinger equation that describes this interaction can be casted in 
a form closely resembling electron-scatteriag from a vibrating ionic diatomic molecule, which in turn can be solved with the LA method. We shall only present a brief overview of the general concepts; an exhaustive treatment and comprehensive bibliographies appear in the book by Mittleman ${ }^{29}$, the review article by Gavrila ${ }^{32}$, and the papers by Csanak and Collins ${ }^{27 .}{ }^{28}$.

The time-dependent (TD) Schroedinger equation in atomic units in the $\mathbf{E} \cdot \mathbf{r}$ gauge that describes the interaction of an electron with an infinitely massive proton and with a temporally-varying electric field has the form ${ }^{29,31,32}$

$$
\left[T(\mathbf{r})+V_{\mathbf{p}}(\mathbf{r})+V_{E}(\mathbf{r} \mid t)\right] \psi(\mathbf{r} \mid t)=i \frac{\partial}{\partial t} \psi(\mathbf{r} \mid t)
$$

where

$$
\begin{gathered}
T(\mathbf{r}) \equiv-\frac{1}{2} \nabla^{2} \\
V_{p}(\mathbf{r}) \equiv-1 / \mathbf{r}, \\
V_{E}(\mathbf{r} \mid t) \equiv \mathbf{r} \cdot \mathbf{E}(t) .
\end{gathered}
$$

The first term $T$ represents the kinetic energy operator for the electron; the second term, the electrostatic interaction of the electron and proton. The final term, in which we have made the usual dipole approximation to the radiation field, gives the interaction of the electron with the electric field $\mathbf{E}(t)$. For the laser configurations and intensities under consideration, we may neglect the magnetic component and the spatial dependence of the electric field. We usually further simplify by taking the electric field as purely oscillatory

$$
\mathrm{E}(t)=\mathrm{E}_{0} \cos (\omega t)
$$

with $\left|\mathbf{E}_{0}\right|$ the amplitude and $\omega$ the frequency. This condition restricts attention to cases in which the laser pulse is long compared to the ramp times although this restriction can be lifted by treating multimode fields. With these caveats, the Schroedinger equation assumes a particularly familiar form, being the starting point for a TD perturbative treatment of photoionization of hydrogen. However, for our case, the electric field of the laser becomes comparable to or greater than the field binding the electron to the proton. A perturbative treatment of the radiation field becomes inappropriate. We therefore seek solutions to Eq.(23) directly.

Before embarking upon this daunting task, we explore certain limiting cases of Eq.(23). If we neglect $V_{E}$, the field terms, we recover the usual, but mundane, Schroedinger equation for an electron scattering from a proton or for an hydrogen atom. The cross section and various $T$-matrix elements show no structure for such collisional events. An analytical solution arises in terms of Coulomb functions for the continuum and hydrogenic functions for the bound states. On the other hand, if we drop the electrostatic term $V_{p}$, we obtain a Schroedinger equation describing the motion of a free electron in an oscillating electrical field. Somewhat surprisingly, this equation too has an analytical solution, termed a Klein-Volkov state ${ }^{34}$. If we form a well-defined gaussian wavepacket from these states, we find some rather interesting properties. First, the center of the packet moves as a classical electron in such an oscillating field

$$
\mathbf{r}(t)=\alpha(t)=\alpha_{0} \cos (\omega t),
$$

where $\alpha_{0}$ is the classical displacement $\left|\alpha_{0}\right|=E_{0} / \omega^{2}$. The packet quivers about the path of a freely-moving electron with an amplitude equal to $\left|\alpha_{0}\right|$. Second, the packet spreads, 
Having established the basic limiting cases, we now coscentrate on a full solution of Eq.(23). Many approaches have been developed for directly solving the TD Schroedinger equation $^{33}$. However, we take a different tact and convert to a time-independent(TI) form that can be solved by standard electron-molecule techniques. We begin by making a transformation due to Kramers and Henneberger ${ }^{30}$ from the laboratory frame to a frame tied to the oscillating electron. In the frame of the electron, the proton appears to oscilliate with frequency $\omega$ and amplitude $\left|\alpha_{0}\right|$, and the effects of the field only appear implicitly. The interaction becomes simply electrostatic in terms of the instantaneous relative position of the two particles. In a more formal sense, the frame transformation is effected through a simple displacement operator

$$
\Omega(\mathbf{r} \mid t)=\exp [-\alpha(t) \cdot \nabla]
$$

with $\nabla$ the usual gradient operator and $\alpha(t)$ given by Eq.(28). Applying this transformation to Eq.(23) yields a TD Schroedinger equation in the Kramers-Henneberger(KH) frame

$$
\left[-\frac{1}{2} \nabla^{2}+V_{p}(\mathbf{r}+\alpha(t))\right] \Phi(\mathbf{r} \mid t)=i \frac{\partial}{\partial t} \Phi(\mathbf{r} \mid t)
$$

where

$$
V_{p}(\mathbf{r}+\alpha(t))=\frac{-1}{|\mathbf{r}+\alpha(t)|}
$$

The transformation, as advertised, has the effect of replacing $\mathbf{r}$ with $\mathbf{r}+\alpha(t)$. We still have a TD equation; however, the field term has been subsumed into the potential. In the KH frame, an electron approaches a charge oscillating with the field frequency remenisent of scattering from a vibrating molecule.

We convert to a TI form by invoking the usual Floquet ansatz. For a purely periodic potential of frequency $\omega$, the solution of Eq.(30) has the general form

$$
\Phi(\mathbf{r} \mid t)=\exp [-i E t] \Phi_{p}(\mathbf{r} \mid t)
$$

where $E$ is a quasi-energy associated with the electron and $\Phi_{p}$ is a periodic function. Since $\Phi_{p}$ has periodic form, we can expand in terms of a Fourier series as

$$
\Phi_{p}(\mathbf{r} \mid t)=\sum_{n=-\infty}^{\infty} \phi_{n}(\mathbf{r}) e^{-i n \omega t}
$$

Substituting Eqs (32-33) into (30), multiplying through by $\exp \left[i n^{\prime} \omega t\right]$, integrating over a period $[T=2 \pi / \omega]$ of the field, and using the relationship

$$
T^{-1} \int_{-T / 2}^{T / 2} \exp \left[i\left(n-n^{\prime}\right) \omega t\right] d t=\delta_{n n^{\prime}}
$$

we find the following TI equation:

$$
\left[-\frac{1}{2} \nabla^{2}-E_{n}\right] \phi_{n}(\mathbf{r})=-\sum_{n^{\prime}} V_{n m^{\prime}}\left(\alpha_{0} \mid \mathbf{r}\right) \phi_{n}(\mathbf{r}),
$$

where $\mathrm{E}_{\mathrm{n}} \equiv \mathrm{E}+\mathrm{n} \omega$, and

$$
V_{n n^{\prime}}\left(\alpha_{0} \mid \mathbf{r}\right)=T^{-1} \int_{-T / 2}^{T / 2} V(\mathbf{r}+\alpha(t)) \exp \left[i\left(n-n^{\prime}\right) \omega t\right] d t
$$


We have reduced the solution of the TD Schroedinger equation to a form [Eq.(35)] closely resembling a normal coupled channel scattering problem. We can further explicate this point by observing the boundary conditions of the channel functions of Eq.(33). As $r$ becomes large, the potential tends to a simple Coulombic form since $|\alpha \mathrm{o}|$ has a prescribed finite value

$$
\lim _{\mathbf{r} \rightarrow \infty}\left|r+\alpha_{0} \cos (\omega t)\right|^{-1} \rightarrow \mathbf{r}^{-1} .
$$

Therefore, the channel functions go to linear combinations of regular and irregular Coulomb functions just as in standard electron scattering from an ion. This characterizes one major advantage of the $\mathrm{KH}$ frame over the $\mathbf{E} \cdot \mathbf{r}[\mathrm{Eq} .(23)]$ or $\mathbf{p} \cdot \mathbf{A}$ formulations. For the latter two cases, the electric field lingers into the asymptotic realm making the boundary conditions more complicated. One other interesting point involves the potential $V_{\text {rur }}\left(\alpha_{0} \mid \mathrm{r}\right)$, which has singularities at $\mathbf{r}= \pm \alpha_{0}$. Although the order of the singularities differ, the form closely resembles the two-center potential encountered in electron scattering from a diatomic molecule. To reinforce this similarity, we display in Fig. 1 the single-center radial expansion coefficients of the elastic term $V_{00}$. In this analogy, the classical displacement $\alpha_{0}$ assumes the role of the internuclear separation $\mathrm{R}$ in the molecule. Finally, since the electric field has been treated classically, we have not mentioned photons. However, we intuitively identify and can formally associate the transition from the $n$ to $n^{\prime}$ Fourier states as the emission or absorption of $n-n^{\prime}$ photons. The equal spacing between Fourier states again reminds us of vibrational excitation. All in all, we find this analogy between the the systems very strong. To solve Eq.(35), we can either apply a single-center expansion or a fully numerical solution by the grid techniques described above. As an example, we make a partial-wave expansion as

$$
\phi_{n}(\mathbf{r})=\sum_{\ell_{n}=0}^{\infty} \sum_{m_{n}=-\ell_{n}}^{\ell_{n}} r^{-1} f_{n \ell_{n} m_{n}}(r) Y_{\ell_{n} m_{n}}(\hat{r}),
$$

with $Y_{l m}$ as spherical harmonic of order $(\ell, m)$. By substituting Eq.(38) into Eq.(35), multiplying through by $Y_{\ell_{n^{\prime}} m_{n^{\prime}}}(\hat{r})^{*}$, integrating over angles, and using the selection rules for spherical harmonics, we obtain

$$
\left[\frac{d^{2}}{d r^{2}}-\frac{\ell_{n}\left(\ell_{n}+1\right)}{r^{2}}+k_{\Gamma}^{2}\right] f_{\Gamma}(r)=\sum_{\Gamma \Gamma^{\prime}} U_{\Gamma \Gamma^{\prime}}(r) f_{r^{\prime}}(r)
$$

where

$$
\begin{gathered}
\Gamma \equiv\left(n \ell_{n}, m_{n}\right), \\
k_{\Gamma}^{2} \equiv 2 E_{n}, \text { and } \\
U_{\Gamma \Gamma^{\prime}}(r)=\int Y_{\ell_{n^{\prime}} m_{n^{\prime}}}(\hat{r}) U_{n n^{\prime}}\left(\boldsymbol{\alpha}_{\mathrm{o}}, \mathbf{r}\right) Y_{\ell_{n} m_{n}}(\hat{r}) d \hat{r}
\end{gathered}
$$

We can now apply the usual linear-algebraic prescription directly to Eq.(39).

\section{APPLICATIONS:}


a stiong temporal electric field. The basic equations governing this process have been briefly reviewed in the previous section. In the absence of the laser field, the cross section and T-matrices as a function of electron energy exhibit no structure since the simplicity of the compound system prohibits Feshbach or shape resonances. However, as demonstrated in Fig.2, introducing the field leads to numerous Feshbach-like resonances in the elastic T-matrix element $\mathrm{T}(0,2 \mid 0,2)$ for a photon energy of $7.35 \mathrm{eV}(.27 \mathrm{au})$ and field strength of $0.0207 \mathrm{au}\left(1.5 \times 10^{13} \mathrm{~W} / \mathrm{cm}^{2}\right)$. The origin of these capture-escape resonances stems from a multiphoton process. The electron emits n photons and falls to an energy level very close to a bound state of the composite system, the hydrogen atom. This compound resonance state has a much longer lifetime due to its temporary trapping in a bound state. In order to conserve energy, the electron must absorb $n$ photons and return to the continuum. The lowest resonance state in Fig.2 corresponds to a twophoton process resonant with the 18 bound state of $H$. The higher resonances involve a single photon with the compound states of the $n=2,3$, and 4 levels. This second has a infinite number of members engaged with the hydrogenic Rydberg series. Inelastic processes such as Bremstralung also occur due to unequal absorptions and emissions. We can also extract multiphoton ionization rates by determining the resonance widths near the appropriate bound state(negative $\mathrm{E}$ ).

\subsection{Collisions of Electrons with $\mathrm{H}_{2}^{+}$}

As another illustration of the applicability of the LAM to a wide variety of phenomena, we describe the scattering of electrons from the hydrogen molecular ion $\mathrm{H}_{2}^{+}$in the regime strongly dominated by autoionizing resonances. While this seems a rather simple sytem, very large-scale calculations, involving many-state close-coupling formulations, are required to obtain accurate scattering information. We treat the basic collision process:

$$
e^{-}+H_{2}^{+} \rightarrow H_{2}^{*} \rightarrow e^{-}+H_{2}^{+}
$$

in which the incident electron becomes trapped in a two-electron excited state of the composite $\mathrm{H}_{2}$ molecule and subsequently decays to the continuum. This process manifests itself as a strong signature in the basic collisonal cross section at an energy near an excited state of the compound system. Such resonances provide intricate tests of methods as well as considerable enhancement in the collisional process.

As an example, we shall concentrate on the energy region just below the first excitation threshold of the ion. The lowest lying states of $\mathrm{H}_{2}^{+}$have symmetries: $1 \sigma_{g}, 1 \sigma_{u}$, $1 \pi_{u}{ }^{+}$, and $1 \pi_{u}{ }^{-}$. We use a generic symbol $k$ for the channel energies, but we recognize that for a given total energy, the wavenumber for each channel will be different. For a choice of total symmetry of ${ }^{1} \Pi_{u}$, the corresponding continuum states are: $k \pi_{u}^{+}, k \pi_{g}^{+}, k \sigma_{g}$, and $\mathrm{k} \delta_{g}^{+}$respectively. A series of bound states of the neutral molecule converge on the ionization thresholds of the ionic system. For example, as the principal quantum number $\mathrm{n}$ increases, the series $1 \sigma_{u} \mathrm{n} \pi_{g}^{+}$has a limit of a bound electron in the $1 \sigma_{u}$ state of $\mathrm{H}_{2}^{+}$and a continuum $\mathrm{k} \pi_{g}^{+}$electron. Similarly the series $1 \pi_{u}^{+} \mathrm{n} \sigma_{g}$ and $1 \pi_{u}^{-} \mathrm{n} \delta_{g}^{+}$converge on the $1 \pi_{u}^{ \pm}$. An electron impinging on the ion at an energy just below the $1 \sigma_{u}$ threshold can easily be trapped in one of these two-electron excited states of $\mathrm{H}_{2}$, producing resonance structure in the cross section. At large internuclear distances, these series remain well separated with those converging on the $\pi_{u}^{ \pm}$thresholds lying energetically above the $1 \sigma_{u}$ ionic level. However, as we decrease $\mathrm{R}$, the lowest resonance states of the $1 \pi_{u}^{+} n \sigma_{g}$ push below the $1 \sigma_{u}$ threshold and begin to interact or mix with the higher-lying doubly-excited states of the $1 \sigma_{u} n \pi_{u}^{+}$series. This interference among the resonance series produces interesting 
effects in the positions and widths(lifetimes) of these levels. For the positions of the lowest few resonances, we note a behavior indicative of curve crossings in molecular systems. At the $\mathrm{R}$ value at which the the lowest-lying state of the higher series begins to cross resonance states of the lower series, we observe a distinct avoided crossing, indicative of the changing character of the resonance. Avoided crossings of electronic states of the same symmetry are well known for bound states but this marked the first quantitative study of the corresponding phenomenon for continuum levels. In Fig.3, we display the width as a function of $R$ for the lowest three resonances in ${ }^{1} \Pi_{u}$ symmetry . For large $\mathrm{R}$, the third lowest resonance has distictly $1 \sigma_{u} 3 \pi_{g}^{+}$character and has a small width. As the $1 \pi_{u}^{+} 2 \sigma_{g}$ state begins to near this level, we note an enhanced feature in the width, characteristic of strong interfrence between the states. Once the intruder state has passed the $1 \sigma_{u} 3 \pi_{g}^{+}$level, the width returns to its original character. We note similar behavior for the crossing of the $1 \sigma_{u} 2 \pi_{g}^{+}$level. For very small internuclear distances, we observe a complete reversal with the first state of the upper series now becoming the lowest resonance. The actual interactions are more complicated, giving rise to the structure of the widths. To obtain reliable values for the resonance parameters, we had to employ an eight-state close-coupling expansion. For a single-center expansion of four partial waves per state and a mesh of one hundred radial points, the LA matrices reached orders of several thousand. Such large, non-sparse matrices were easily treated using the variation/iteration approach discussed in section 3.2. Whether these effects can be observed in synchrotron studies will depend on the development of an experimental capability to excite the initial $\mathrm{H}_{2}$ to a vibrational level high enough to access the FranckCondon region of the resonance. In addition, the observation of adiabatic as opposed to diabatic behavior of the electronic states involved in the crossing, can be influenced by other dynamic considerations. In any event, we hope this has demonstrated that even such simple systems as $\mathrm{H}_{2}$ still have much to offer for theorists to contemplate. 


\section{Refererices}

1 Kopal, Z., Numerical Analysis $2^{\text {nd }}$ ed., (John Wiley and Sons, New York, 1961).

2 Robertson, H. H., Proc. Cambridge Phil. Soc. 52, 535 (1956)

3 Dongarra, J. J., Moler, C. B., Bunch, J. R. and Stewart, G. W., LINPACK Users' Guide, (SIAM, Philadelphia/1979)

4 Faddeev, D. K. and Faddeeva, V. N., Computational Methods of Linear Algebra (W. H. Freeman and Co., San Francisco and London, 1963)

5 Simply stated the problem is that the numerical integration process becomes contaminated by the exponentially growing, unwanted other solution to the second order set of equations. The undesired solutions may be eliminated during the integration process by matrix factorization procedures which take $N^{3}$ operations.

6 Seaton, M. J., J. Phys. B 7, 1817 (1974)

7 Crees, M. A. and Moores, D. L., J. Phys. B 8, L195 (1975)

${ }^{8}$ Schneider, B. I. and Collins, L. A., Phys. Rev. A24, 1264 (1981)

${ }^{9}$ Collins, L. A. and Schneider, B. I., Phys. Rev. A24, 2387 (1981)

${ }^{10}$ Schneider, B. I. and Collins, L. A., Phys. Rev. A27, 2847 (1983)

11 Schneider, B. I. and Collins, L. A., Phys. Rev. A33, 2970 (1986)

12 Schneider, B. I. and Collins, L. A., Comput. Phys. Comm. 53, 381 (1989)

13 Schneider, B. I. and Collins, L. A., Phys. Rev. A33, 2982 (1986)

14 Collins, L. A. and Schneider, B. I., J. Phys. B 17, L235 (1984)

15 Collins, L. A. and Schneider, B. I., Phys. Rev. A34, 1564 (1986)

${ }^{16}$ Schneider, B. I. and Collins, L. A., Comp. Phys. Reports 10, 51 (1989)

17 See the articles on the $\mathrm{R}$-matrix and Kohn variational methods in this volume.

18 Comput. Phys. Comm. 53, 381 (1989) was devoted entirely to the use of iterative methods in chemistry and physics. It is an excellent starting point and contains most of the important references.

19 Chase, D. M., Phys. Rev. 104, 838 (1956)

20 Temkin, A. and Vasavada, K. V., Phys. Rev. 160, 109 (1967)

${ }^{21}$ Feshbach, H, Ann. Phys. 5, 357 (1958)

22 Light, J. C. and Walker, R. B., J. Chem. Phys. 65 ,4272 (1976)

${ }^{23}$ Schneider, B. I. and Walker, R. B., J. Chem. Phys. 70, 2466 (1979)

${ }^{24}$ Noble, C. J. and Nesbet, R. K., Comput. Phys: Comm. 33, 399 ( 1984 )

25 Becke, A. D., J. Chem. Phys. 88, 2547 (1988)

${ }^{26}$ Becke, A. D. and Dickson, R. M., J. Chem. Phys. 89, 2993 (1988)

27 Csanak, G and Collins, L. A. Phys. Rev. 47, 3240 (1993).

${ }^{28}$ Collins, L. A. and Csanak, G., Phys. Rev. 44, R5343 (1991).

${ }^{29}$ Mittleman, M. H., Introduction to the Theory of Laser-Atom Interactions, $2^{\text {nd }}$ ed., (Plenum, New York, 1993).

${ }^{30}$ Henneberger, W. C., Phys. Rev. Lett. 21, 838 (1968).

31 Mittleman, M. H., Phys. Rev. A42, 5645 (1990).

32 Atoms in Intense Laser Fields, ed. Gavrila, M. (Academic Press, New York, 1992).

33 Time-Dependent Methods for Quantum Dynamics, ed. Kulander, K., Comp. Phys. Comm. 63 (1991).

${ }^{34}$ Volkov, D. M., Z. Phys. 94, 250(1935). 